\title{
Identifying the information for the visual perception of relative phase
}

\author{
ANDREW D. WILSON \\ University of Aberdeen, Aberdeen, Scotland \\ AND \\ GeOFFrey P. BINGHAM \\ Indiana University, Bloomington, Indiana
}

\begin{abstract}
The production and perception of coordinated rhythmic movement are very specifically structured. For production and perception, $0^{\circ}$ mean relative phase is stable, $180^{\circ}$ is less stable, and no other state is stable without training. It has been hypothesized that perceptual stability characteristics underpin the movement stability characteristics, which has led to the development of a phase-driven oscillator model (e.g., Bingham, 2004a, 2004b). In the present study, a novel perturbation method was used to explore the identity of the perceptual information being used in rhythmic movement tasks. In the three conditions, relative position, relative speed, and frequency (variables motivated by the model) were selectively perturbed. Ten participants performed a judgment task to identify $0^{\circ}$ or $180^{\circ}$ under these perturbation conditions, and 8 participants who had been trained to visually discriminate $90^{\circ}$ performed the task with perturbed $90^{\circ}$ displays. Discrimination of $0^{\circ}$ and $180^{\circ}$ was unperturbed in 7 out of the 10 participants, but discrimination of $90^{\circ}$ was completely disrupted by the position perturbation and was made noisy by the frequency perturbation. We concluded that (1) the information used by most observers to perceive relative phase at $0^{\circ}$ and $180^{\circ}$ was relative direction and (2) becoming an expert perceiver of $90^{\circ}$ entails learning a new variable composed of position and speed.
\end{abstract}

Coordinated rhythmic movement is organized in a highly characteristic fashion (described by Haken, Kelso, \& Bunz, 1985, with the so-called HKB model); $0^{\circ}$ mean relative phase (two oscillators doing the same thing at the same time) and $180^{\circ}$ (two oscillators doing the opposite thing at the same time) are the only two stable modes, with $0^{\circ}$ more stable than $180^{\circ}$ (as frequency is increased, there is a tendency to transition from $180^{\circ}$ to $0^{\circ}$, but never the other way). A mean relative phase of $90^{\circ}$ (halfway between $0^{\circ}$ and $180^{\circ}$ ) is maximally unstable, although it can be learned (e.g., Zanone \& Kelso, 1992a). The issue at hand is why this class of movement should be organized the way that it is; the hypothesis is that this organization is rooted in the perceptual information used to perform the task. This hypothesis is based on observations that the phenomena persist when the coupling is between people (Schmidt, Carello, \& Turvey, 1990; Temprado, Swinnen, Carson, Tourment, \& Laurent, 2003) or between a person and a display (Buekers, Bogaerts, Swinnen, \& Helsen, 2000; Wilson, Collins, \& Bingham, 2005b; Wimmers, Beek, \& van Wieringen, 1992). This and other research inspired a perception-action model (Bingham, 2001, 2004a, 2004b), which describes a task dynamic for the production of coordinated rhythmic movement that comprises both action and information components. The model makes predictions about the identity of the informational component that causes the movement pattern, and the present study is a detailed psychophysical test of these predictions.

There have been two streams of research in which the hypothesis that the coordinated rhythmic movement pattern has a perceptual basis has been investigated. The first stream has entailed participants' making judgments about coordinated rhythmic movements, presented either visually (Bingham, 2004a; Bingham, Schmidt, \& Zaal, 1999; Bingham, Zaal, Shull, \& Collins, 2001; Zaal, Bingham, $\&$ Schmidt, 2000) or proprioceptively (Wilson, Bingham, $\&$ Craig, 2003). The judgment data have mirrored the movement pattern: Judgments of $90^{\circ}$ were highly variable, those of $180^{\circ}$ less so, and those of $0^{\circ}$ hardly at all. This suggested that the pattern emerges in movement as a result of how well information about the conditions is detected. In the second stream, this has been tested by manipulating the perceptual feedback used to control a coordinated movement and measuring how movement stability changes in response (Bogaerts, Buekers, Zaal, \& Swinnen, 2003; Mechsner, Kerzel, Knoblich, \& Prinz, 2001; Wilson et al., 2005b). Movement stability varied as a function of the relative phase of the feedback, rather than the relative phase of the movement. Non- $0^{\circ}$ movements are,

A. D. Wilson, andrew.wilson@abdn.ac.uk 
therefore, not intrinsically unstable; if the participant can readily discriminate the information used to perform the task, this stable perception allows for stable movement. These experimental manipulations suggest that movement stability is largely a function of perceptual stability. ${ }^{1}$ The question remains, what is the identity of the perceptual information being used?

Bingham (2004a) reported a series of experiments that identified the characteristics of the information. First, participants judged phase variability at five mean relative phases, each with four levels of added phase variability (from $0^{\circ}$ to $15^{\circ}$ phase standard deviation), at three frequencies. Phase variability was clearly discriminated only at $0^{\circ}$, and what discrimination there was at $180^{\circ}$ disappeared with an increase in frequency. Non- $0^{\circ}$ relative phases were judged to be intrinsically variable, $90^{\circ}$ maximally so (see also Bingham et al., 2001). A second experiment placed the added variability at different locations along the spatial trajectory of the displays (aligned with peak velocity, peak amplitude, or both peak velocity and peak amplitude or distributed throughout the trajectory). Variability was detected identically at all points for $0^{\circ}$; at $180^{\circ}$, variability was still detected at all points in the trajectory, but not equally (detection was poor at peak velocity). Bingham (2004b) concluded that relative speed functioned as a noise term, affecting the participants' ability to resolve the underlying information. $\mathrm{He}$ also concluded that the information itself was present at all points in the trajectory and that, under these conditions, phase perception could, therefore, be continuous.

On the basis of these results, Bingham (2001, 2004a, 2004b) proposed a perception-action dynamical systems model of coordinated rhythmic movement, in which two nonlinearly damped mass-spring oscillators are coordinated via a perceptual coupling function; each mass-spring is driven by the perceived phase of the other mass-spring, modified by perceived relative phase. Constrained by the empirical data described above, the model predicted that the information for perceived relative phase is the relative direction of motion of the two oscillators, the detection of which is modulated by relative speed. These are simply the two elements (direction and magnitude, respectively) of the vector quantity relative velocity, the first temporal derivative of relative position. This predicts the characteristic phenomena: $0^{\circ}$ and $180^{\circ}$ are distinctive because they are the mean relative phases at which the relative directions are always the same $\left(0^{\circ}\right)$ or always different $\left(180^{\circ}\right)$; $90^{\circ}$ is the point at which the relative direction is the same half the time and different the other half of the timethat is, maximally variable. The $0^{\circ}$ mean relative phase is stable because the relative speed is zero and the relative directions (which are consistent and consistently the same) are, therefore, easily resolved; $180^{\circ}$ is less stable because the relative speed ranges from zero to maximally different and the relative directions (which are still consistent but now consistently different) are, therefore, more difficult to discriminate (because of the nonzero relative speeds). Relative direction is hardest to detect at $90^{\circ}$ because it is maximally variable and also because relative speed is always nonzero.
A crucial role for relative direction has been implicit in other research for some time. Wimmers et al. (1992) showed that when the movements to be coordinated were orthogonal to each other, performance was uniformly less stable, but with no tendency to transition from $180^{\circ}$ to $0^{\circ}$. They tested both possible mappings (the top of a vertically moving signal corresponded, on different trials, to either the left or the right of a horizontal limb movement) and found no difference and no preference for in-phase (e.g., top/left or top/right) movement. It is technically possible to talk about a relative phase between two orthogonally moving oscillators, but this empirical result (and indeed, the existence of two mappings) shows that the mapping is arbitrary; left can equally map to top or bottom. Relative phase is unambiguously defined only when the oscillators are moving in parallel to one another (or with significant parallel components of motion). This, by hypothesis, is because relative direction is defined only in these cases.

More direct evidence has come from Bogaerts et al. (2003). They had people perform cyclical drawing movements with both hands, and the movements were either parallel or orthogonal to each other. Orthogonal movements were again (as in Wimmers et al., 1992) less stable than parallel movements. When visual feedback of the task was altered so that the orthogonal movements produced parallel motion on a monitor, the orthogonal movements were stabilized. The biggest improvement was seen with orthogonal/antiphase movement while transformed feedback depicting parallel antiphase motion was viewed. The authors cite this as demonstrating how important the parallel component of motion is to forming a clearly perceived ("perceptually coherent") form, which can then be used to produce stable coordinated movements. The fact that parallel motion was more important than isodirectional motion in stabilizing movement suggests that parallel motion is a prerequisite for movement coordination; relative direction must be definable for the perception-action system to begin coordination. Wilson, Collins, and Bingham (2005a) found that tracking a linear (side-to-side) motion with a circular action was qualitatively the same as tracking it with a linear action. The circular motion added an orthogonal component (top to bottom) to the participants' movement that had no phase-specific effects on movement stability, suggesting that the relative phase between that component and the side-to-side motion being tracked did not influence the stability of the coordination. Relative speed was still defined across orthogonal motions; the additional component therefore added noise to the task, but uniformly at all mean relative phases.

The hypothesis that relative direction is the information used to detect relative phase also predicts numerous characteristics of rhythmic movement coordination. First, it predicts the basic movement phenomena. Second, it is a variable that both vision and proprioception can detect, which accounts for the replication of the judgment results in these two modalities (see, e.g., Zaal et al., 2000, and Wilson et al., 2003, respectively). Third, it also predicts the transfer of learning seen in Zanone and Kelso (1992a, 1992b, 1997). Learning at $90^{\circ}$ transferred only to the so-called symmetry partner of $270^{\circ}$, which is so 
called because relative direction is the same half the time and different half the time in both cases (ignoring which oscillator leads and which follows). Similarly, learning at $135^{\circ}$ generalized only to its symmetry partner, $225^{\circ}$. From the present perspective, learning does not transfer, per se, from $90^{\circ}$ to $270^{\circ}$ or from $135^{\circ}$ to $225^{\circ}$; rather, as far as relative direction is concerned, the symmetry partners are identical coordinations. Fourth, the hypothesis that relative direction is the information being used also allows for the fact that learning generalizes across limbs (Kelso \& Zanone, 2002); learning has nothing to do with the oscillator per se (whether it is an arm or a leg), but only with the motion of the oscillator. Finally, relative direction unifies the results from Fontaine, Lee, and Swinnen (1997) and Wenderoth, Bock, and Krohn (2002) with the results from the rest of the learning literature. Both of these studies showed (contra Zanone \& Kelso, 1994) that relative phases close to $0^{\circ}$ (e.g., $30^{\circ}$ ) were easier to learn than relative phases close to $180^{\circ}$ (e.g., $150^{\circ}$ ). Perception of relative direction, conditioned on relative speed, predicts that the region around $0^{\circ}$ should be very clearly and finely resolved; $30^{\circ}$ will, perceptually, be much more distinct from $0^{\circ}$ than $150^{\circ}$ is from $180^{\circ}$, making it easier to learn. Also, learning rate varied inversely with proportion of time the oscillators spent moving in the same direction in Wenderoth et al.; in other words, the easier it is to detect the information, the easier it is to learn it. Overall, the circumstantial case for what information is used is strong, but it is still circumstantial. The present experiments were therefore designed to explicitly test what information underpins judgments of coordinated rhythmic movement. To do this, we employed a perturbation method.

\section{Perturbation Method}

The phase-driven oscillator model is a dynamical system that contains both perceptual and motor components. A common way to explore such a system is the perturbation experiment. The premise of this experiment is the idea that a given perception-action task uses specific informational and motor components and explicitly does not use others. Formation of a stable perception-action system requires that the relevant components temporarily become functionally "walled off" from other components. This softly assembled task-specific device (Bingham, 1988) becomes relatively impervious to irrelevant distractions, allowing the behavior in question to be accomplished stably and reliably for the duration of the task. Perturbing an information variable that the system is currently ignoring will, therefore, have little or no effect on the behavior, whereas perturbing a variable required for the task will interfere with the performance of the task, in a manner specific to the role that the variable plays. A good example of the former entailment can be found in Mechsner and Knoblich (2004). They made fingers being coordinated more visually salient by adding colored cuffs to the fingers, but this manipulation had no effect whatsoever on movement stability. Color is an example of an information variable to which the coordinated rhythmic movement task-specific device is insensitive; it is functionally irrelevant to the task, and hence, performance was impervious to the manipulation.
An advantage of the perturbation design is that the task can be performed under full-cue conditions, fully representative of the task of interest. Presenting candidate information variables in isolation and measuring thresholds would not be informative about how these are used in the overall task dynamic of coordinated rhythmic movement. Presenting a rhythmic display in which all the information is present but one aspect has been selectively perturbed allows us to investigate whether a variable is even used in the task and, if so, how (i.e., this method provides a measure of both the composition and the organization of the dynamic).

The present experiment was designed to systematically perturb the various possible information variables that might be part of the coordinated rhythmic movement dynamic, and we used the predictions of the phase-driven model to generate both candidate variables and the predicted consequences of perturbing them. First, the model simulates judgments of mean relative phase by integrating the relative direction term over time. Relative phase is, therefore, specified by the proportion of time the oscillators spend moving in the same or the opposite direction. In pilot work, we attempted to perturb this relative direction behavior independently of mean relative phase in two different ways (using a sinusoidal and a square wave spatial path for the dots). Both attempts failed. The sinusoidal displays produced a motion that could be (and clearly was) decomposed by the visual system into the two component sinusoids (cf. Johansson, 1950), nullifying the perturbation. The square waves allowed us to simulate the behavior of relative direction for a given relative phase, but the dots were not, in fact, moving at that relative phase, and the judgment task became nonsensical. By the nature of the task domain, it is impossible to perturb relative direction independently of relative phase; the latter is defined by the former.

We therefore had to proceed by a process of elimination. Although the role of relative direction is clear, there are two other aspects of the dots' motion that people could, in principle, be using to perceive relative phase: relative position and relative speed.

First, it is quite plausible that relative phase perception entails perception of the phases of each individual oscillator and a computation of the difference between them. Phase is computed as an angle in the phase plane (a plot of position on the $x$-axis and speed on the $y$-axis). The origin of a given phase plane is defined by the location and value of peak speed, and this origin defines the frame of reference required to identify the phase of a movement of given amplitude and frequency. Perceptually, phase is a location along the trajectory form (Muchisky \& Bingham, 2002; Wickelgren \& Bingham, 2001, 2004, 2008). This location is specified in the phase-driven model by the current relative proportion of the peak speed. To test whether phase underpins judgments of relative phase, we perturbed both the value and the location of the peak speed; that is, we perturbed the reference frame. This was done independently of relative direction, and therefore, the model predicts that this perturbation should have no effect on judgments of relative phase. 
We perturbed the entire reference frame by randomly altering amplitude on each half-cycle of motion ( perturb position condition). The amplitude on one half-cycle was no longer informative about the amplitude of the next half-cycle, and therefore, neither (1) the location nor (2) the value of the peak speed was specified before the peak was actually achieved and passed. The origin of the phase plane and, hence, the reference frame were, therefore, not specified, and there was no stable information for phase besides relative direction (see Figure 1, left column, for a phase portrait of a perturbed dot and a time series of two dots moving at $90^{\circ}$ mean relative phase under this perturbation).

Next, to control for having perturbed two aspects of the peak speed in the position perturbation, we perturbed only its value and not its position. We perturbed frequency by varying it over the course of a trial (perturb frequency; see Figure 1, center column, which shows a phase portrait of a perturbed dot and a time series of two dots moving at $90^{\circ}$ under this perturbation). The effect of this manipulation was to alter the value of the peak speed on each half-cycle, but not the location at which it was achieved. In this case, observers would be able to see that they were halfway from the endpoint of movement to the peak speed and, thus, would be better able to perceive the locus along the trajectory before the peak was actually reached. Nevertheless, the continuous variation in the frequency (and thus, in the speed) would make the detection of the locus less reliable - that is, noisy.

The second possible source of information about relative phase is the relative speed profile (the pattern of change in the speed difference between the oscillators over the course of a cycle). Different mean relative phases do, indeed, show different relative speed profiles, and so, in principle, relative phase can be specified by relative speed. However, these profiles specify relative phase only for a specific pair of amplitudes. If the two dots in the display are moving at different amplitudes, the relative speed profile at $0^{\circ}$ could now be identical to that for movement at (for instance) $180^{\circ}$ with equal amplitudes. It seems unlikely that a participant would rate $0^{\circ}$ motion to be $180^{\circ}$ motion under such circumstances, and having to account for amplitude in this fashion makes relative speed highly unstable information about relative phase. In addition, the psychophysical evidence described above (Bingham, 2004a) suggests that relative speed is not the information but, instead, makes the detection of the information harder. The model therefore predicts that the speed difference is only a noise term. We tested this by increasing the amplitude of one dot's motion, thereby increasing the magnitude of the relative speed difference ( perturb speed; see Figure 1, right column, for a phase portrait and time series of two dots moving at $90^{\circ}$ under this perturbation).

We carried out these experiments on two different groups. The first experiment was designed to systematically test the predictions of the model. Participants were shown pairs of perturbed displays, one of which was the target phase $\left(0^{\circ}\right.$ or $\left.180^{\circ}\right)$ and one of which was different by some amount, and were told to identify which was $0^{\circ}$ (or $180^{\circ}$ ). The model predicted that all three perturbation conditions would only add noise to performance, because none of the conditions affected relative direction. In the second experiment, this paradigm was used to identify the information the participants had learned to use in a separate experiment in which they had become experts at discriminating $90^{\circ}$ (Wilson \& Bingham, 2008). Recall that learning to move at $90^{\circ}$ generalizes only to the symmetry partner $270^{\circ}$ (Zanone \& Kelso, 1992a, 1992b, 1997). This encapsulation suggested that learning $90^{\circ}$ entails learning to use a novel informational variable, rather than simply getting better at using the variable that was previously poorly detected. If it were the latter, this improved discrimination would be expected to improve performance at $0^{\circ}$ and $180^{\circ}$, and it does not. We therefore predicted that at least one of the perturbations motivated by the model (position, frequency, or speed) would completely disrupt the participants' performance, implicating that variable.

\section{EXPERIMENT 1 Perturb $0^{\circ}$ and $180^{\circ}$}

\section{Method}

\section{Participants}

Ten participants from Indiana University ( 8 of them male, 2 female), 22 to 53 years of age, took part and were paid $\$ 10 / \mathrm{h}$. The experiment was approved by the local IRB and was carried out in accordance with the Declaration of Helsinki.

\section{Apparatus and Design}

Displays were presented using a Dell Optiplex computer and controlled by custom $\mathrm{C}++$ libraries. The monitor was set to a $1,024 \times 768$ pixel resolution with a $60-\mathrm{Hz}$ refresh rate. Viewing distance was not restricted, but all the participants sat approximately $40-50 \mathrm{~cm}$ from the monitor. The participants were free to move their eyes as well.

The participants were tested on three types of perturbation (position, speed, and frequency) at two mean relative phases $\left(0^{\circ}\right.$ and $180^{\circ}$ ), as well as being tested with unperturbed $180^{\circ}$ displays. Each condition was run as a block, and the participants did all seven conditions in a single session.

We did not collect data from these participants in a baseline $0^{\circ}$ task; the task is trivially easy because (in the unperturbed displays) it becomes a judgment of rigid versus nonrigid motion (which people are very good at; Braunstein, Hoffman, \& Pollick, 1990), rather than identification of a relative phase. We tested 4 participants on this task as a control study to confirm this prediction, and all 4 were able to flawlessly pick $0^{\circ}$; debriefing suggested that all of the participants were using rigidity to make the judgment.

\section{Procedure}

Choose $0^{\circ} / \mathbf{1 8 0}^{\circ}$. The choose $0^{\circ}$ and choose $180^{\circ}$ tasks were presented in separate blocks but shared the same design. Each trial consisted of a pair of successively presented stimuli (two dots moving harmonically on the screen at some mean relative phase, with an amplitude of 300 pixels, for $4 \mathrm{sec}$ at $1 \mathrm{~Hz}$ ). One of each pair showed two dots moving at $0^{\circ}$ (or $180^{\circ}$ ), and the other was either the same or different. The task was two-alternative forced choice (2AFC); the participants had to identify which display in the pair was $0^{\circ}$ (choose $0^{\circ}$ task) or $180^{\circ}$ (choose $180^{\circ}$ task). They responded first by pressing the "A" key, second by pressing the "L" key; there was no time constraint, and reaction time was not measured. The same trials were catch trials and were there to provide a measure of response bias. Four different locations were tested (two less than $0^{\circ} / 180^{\circ}$, two greater), and $0^{\circ} / 180^{\circ}$ was either the first or the second display (50:50 split); there were, therefore, nine different trial types (4 different locations $\times 2$ orders, plus the catch trial). Choose $0^{\circ}$ 


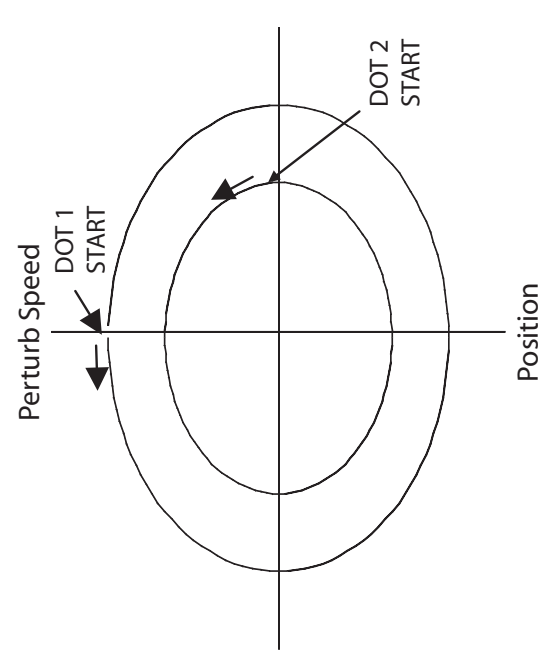

Кұ!ฺำ

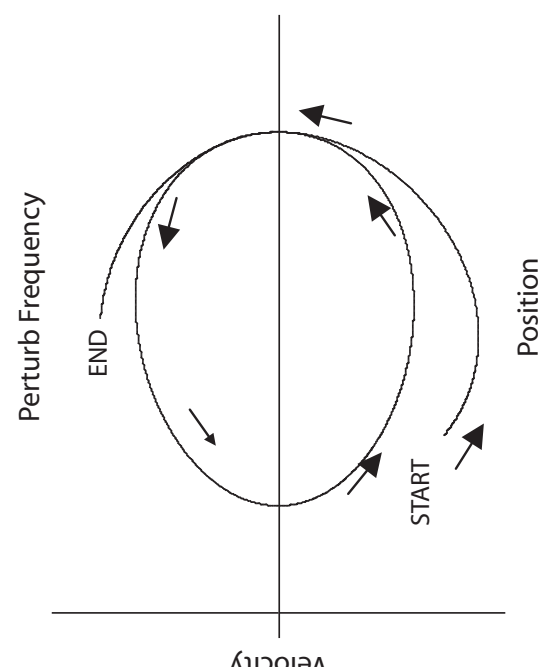

Кұ!ฺ이

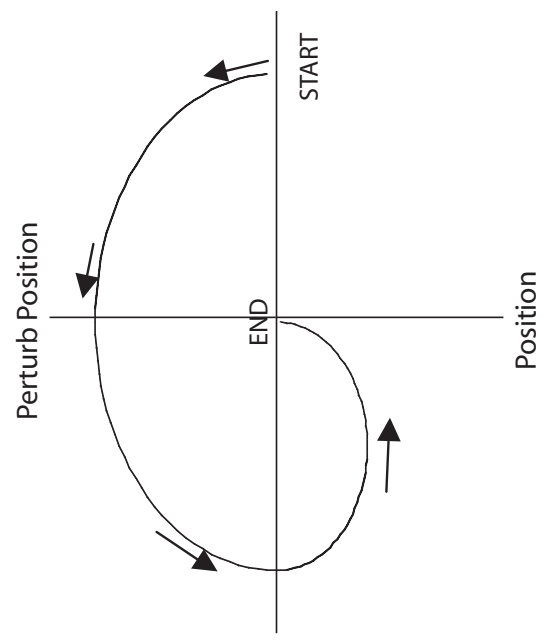

К!ำ이시

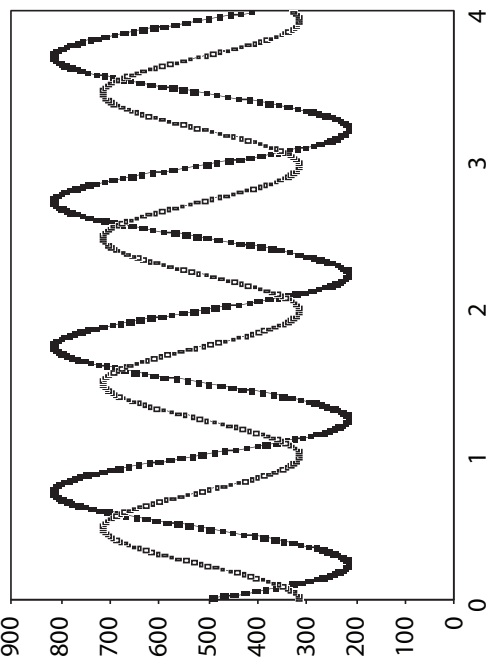

(s|әx!d) uo!n!sod s!x $\forall-X$

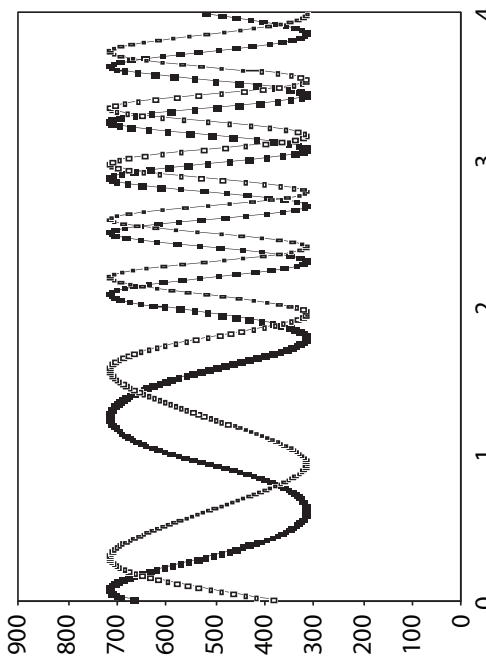

(s|əx!d) uo!?!sod s!x $\forall-X$

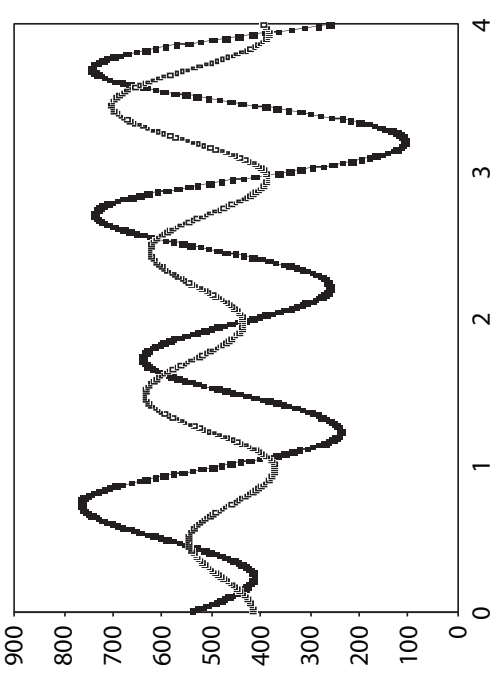

(s|əx!d) uo!!!!sod s!X $\mathrm{X} \forall-X$

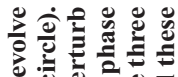

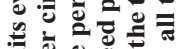

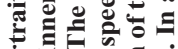

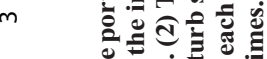

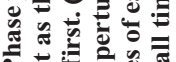

ญू

$\sim$ ฮ

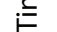

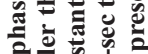

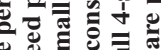

ङ के

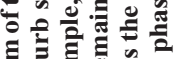

ह छ

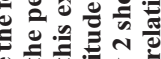

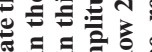

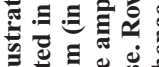

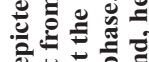

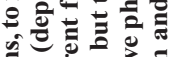

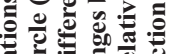

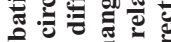
光 츨

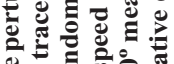

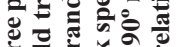

ญ

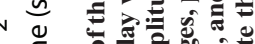

毛 ट⿱㇒日勺

ซ

흐월

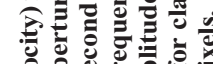

을 氙

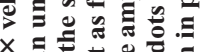

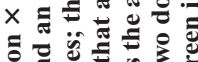
ฮี

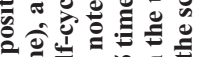

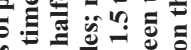

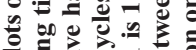

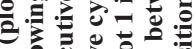
践.

影

¿

인

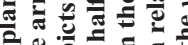

运元

ป

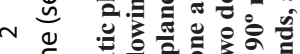

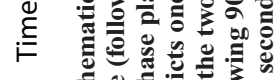

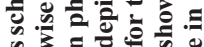

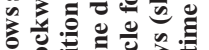

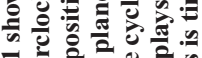

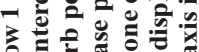

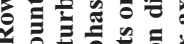

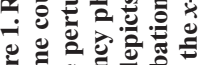

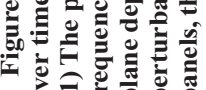


involved discriminations between $0^{\circ}$ and $330^{\circ}, 345^{\circ}, 15^{\circ}$, and $30^{\circ}$. Choose $180^{\circ}$ involved discriminations between $180^{\circ}$ and $150^{\circ}, 165^{\circ}$, $195^{\circ}$, and $210^{\circ}$. These sets were chosen, on the basis of pilot work, to be hard but not at the limit of performance. The participants were presented with five blocks of each task. Each block contained one of each trial type, with display order randomized within blocks. There was no feedback given during these trials; there was, however, a brief practice session that gave examples of $0^{\circ}$ and $180^{\circ}$, as well as four practice trials of the $2 \mathrm{AFC}$ task, with feedback.

\section{Displays}

Perturb position. Perception of relative phase may depend on the detection of the relative positions of the two oscillators within their respective cycles (see Figure 1, left). A stable frame of reference is essential for specifying position with the cycle. Peak speed occurs at the midpoint of each half-cycle, and this origin defines such a stable frame of reference. Without constant amplitude, the location of the midpoint of the half-cycle and, thus, the reference frame keeps changing. An observer would be unable to detect the relative position and, thus, would be unable to detect where along the trajectory form they are (until peak speed actually occurs). The trajectories were therefore perturbed by randomly changing the amplitude of the dots from half-cycle to half-cycle (where a half-cycle is defined as the dots' moving from one side of the screen to the other). On the phase plane, this is equivalent to the dots' moving from one circle to another every half-cycle.

If the amplitudes of the two dots remain identical (i.e., if they change by the same amount each half-cycle), they remain moving in lockstep. The position of the midpoint is successfully perturbed, but at non- $0^{\circ}$ relative phases, there would be two opportunities to detect it, one for each dot. The amplitude of the bottom dot was therefore set to be half the amplitude of the top dot, which meant that the position of the midpoint was always different for the two dots.

The participants performed the choose $0^{\circ}$ and choose $180^{\circ}$ tasks with displays perturbed in this fashion. The model predicts that because this perturbation does nothing to relative direction, there should be no effects of the perturbation on judgments of relative phase.

Perturb frequency. Computation of phase requires that you normalize the data by the frequency (see Figure 1, center). We were therefore interested in whether participants need to perceive frequency in order to perceive phase. We therefore designed a perturbation that made frequency (and hence, the relative speed profile) variable, while keeping amplitude constant. This altered peak speed in each half-cycle. This perturbation should make the specification of the current location along the trajectory form noisier and less reliable (with respect to the speed), but location should still actually be indicated (relative to the [unperturbed] endpoints and midpoint of the displacement).

Frequency was varied over the course of each trial according to the function

$$
\text { frequency }=\text { initialFreq }+ \text { amplitude } * \sin (\text { time }) *(2 * \pi)] \text {. }
$$

This equation was evaluated at each time step, and the new value for frequency was used in computing the positions. Frequency began at $1.25 \mathrm{~Hz}$ and smoothly varied sinusoidally from a minimum of $1.0 \mathrm{~Hz}$ to a maximum of $1.5 \mathrm{~Hz}$. In half the trials, the frequency first decreased and then increased, whereas in the other half, this was reversed. There were no differences in performance for these two display types, so all the analyses combined these data.

Perturb speed. Relative speed is simply the magnitude of the speed difference between the two moving dots. Harmonic motion at different mean relative phases shows different relative speed profiles (see Figure 1, right); if the two dots are at $0^{\circ}$ mean relative phase (with identical frequency and amplitude), the relative speed is always zero, whereas at $180^{\circ}$, relative speed varies from zero (at the end points) to a maximum (at the midpoint, where the two dots are both at peak velocity but heading in opposite directions). This might, therefore, be used to specify relative phase. The model, however, predicts only that the speed difference contributes a noise term that acts on the detection of relative direction and is not itself the information. To test this, we perturbed relative speed by an amplitude manipulation. One oscillator (the top dot) moved at 1.5 times the amplitude of the bottom dot, the amplitude of which was identical to the unperturbed displays. These amplitudes were constant within and between trials. To preserve a global mean relative phase, the oscillator with the larger amplitude had to move faster. The magnitude of the speed difference was, therefore, higher than that in the unperturbed displays, all the way through the trajectory - increasing the amount of noise, but increasing it uniformly for all mean relative phases. On the phase portrait, this produces two concentric circles.

\section{Data Analysis}

The data from this task were the frequencies with which the participants responded $0^{\circ}$ first or $180^{\circ}$ first. The frequency data were analyzed with each trial being described by the magnitude of the phase difference. This places data from (for instance) $150^{\circ}-180^{\circ}$ trials and $210^{\circ}-180^{\circ}$ trials at the same point on the axis, specifically -30 (sign indicates that the different trial was shown first). The $x$-axis therefore had five locations: $-30,-15,0$ (the catch trials), 15 , and 30 .

A nominal logistic regression model was fit separately to each participant's data set. The model-fitting procedure estimates two parameters, intercept and slope, as well as confidence intervals for each parameter. A mean regression curve was fit using the parameter estimates. The absolute values of the mean relative phase difference at which the probability was $25 \%$ and $75 \%$ of responding $0^{\circ}$ first or $180^{\circ}$ first was computed from each regression line (by solving for $x$ ) and was averaged to produce a robust estimate of the threshold (distance from the target phase required before the target phase could be reliably identified).

\section{Results and Discussion}

Examination of the individual data revealed that 3 of the 10 participants had been affected at both $0^{\circ}$ and $180^{\circ}$ by the position perturbation (see Figure 2). Their thresholds were more than two standard deviations away from those for the other 7 participants, so their data were excluded from the group analysis. We will discuss their performance, however, because they illustrate two very important points: the scale of a genuine perturbation result (as opposed to being slightly more noisy) and the vital importance of considering individual behavior in a task such as this one, in which there is more than one potential perceptual solution to the problem.

As is shown in Figures 2 and 3, the results were as follows. (1) The position perturbation did statistically elevate thresholds in the choose $0^{\circ}$ task; however, it elevated them only to the same level as that for $180^{\circ}$ under this perturbation. Given that these perturbations do not seem to have completely disrupted performance (as would be expected if the variable being perturbed was being used in the task), why the extra effect at $0^{\circ}$ ? As was described earlier, one relevant way in which $0^{\circ}$ is unique is that when two oscillators are moving precisely at $0^{\circ}$, they define a rigid motion (i.e., the distance between the points does not vary). Because of this, it was pointless to test the baseline $0^{\circ}$ task. It is done by simply comparing rigid versus nonrigid motion in the displays, a task at which human observers are nearly ideal (Braunstein et al., 1990). This rigidity was no longer present in the position perturbation, making the $0^{\circ}$ displays now more like the $180^{\circ}$ displays in this regard. The perturbation added noise to the judgment, but uniquely to $0^{\circ}$. (2) None of the perturbations, therefore, disrupted 


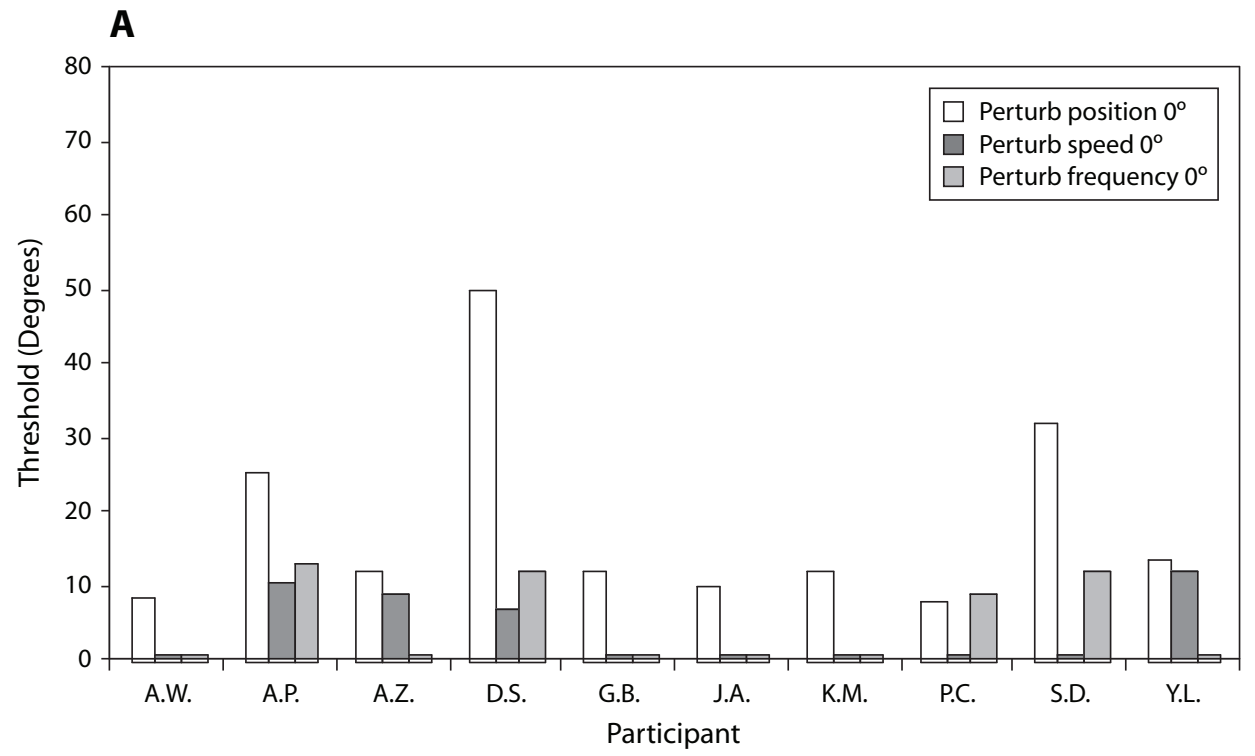

B

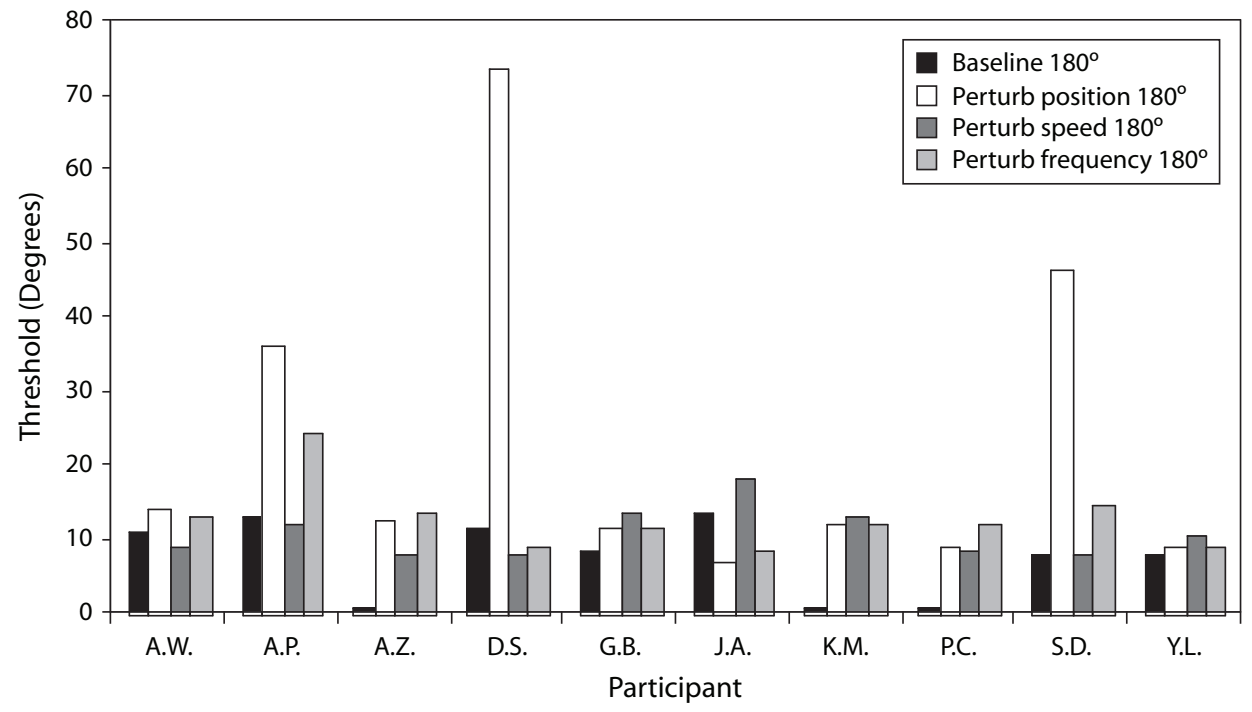

Figure 2. Thresholds for the individual participants in the $0^{\circ}(\mathrm{A})$ and $180^{\circ}(\mathrm{B})$ conditions in Experiment 1. Perturb position is shown using white bars; perturb frequency is shown using dark gray bars; and perturb speed is shown using light gray bars. Panel B includes the baseline $180^{\circ}$ data in black bars (there is no baseline $0^{\circ}$ data; see the text for details). Participants 1,3 , and 9 were completely perturbed at both $0^{\circ}$ and $180^{\circ}$ by the position perturbation, but all other effects were small.

these participants' ability to do the task; the one effect (position, $0^{\circ}$ ) simply made it slightly harder (more noisy). As context, the 3 participants we excluded showed genuine disruption, with thresholds ranging from $25.48^{\circ}$ to $73.81^{\circ}$ (Figure 2). The largest group mean from the choose $0^{\circ}$ or choose $180^{\circ}$ tasks (perturb speed, $180^{\circ}$ ) was $11.61^{\circ}$ and, therefore, not on the order of a genuine perturbation effect. None of these 7 participants, therefore, used relative position or relative speed as the perceptual information for their judgments, and the perturbations simply made detection of the actual information slightly harder.
We performed a repeated measures ANOVA on the mean thresholds for the remaining 7 participants. There were two within-subjects factors: perturbation (three levels: position, speed, or frequency) and phase (two levels: $0^{\circ}$ or $180^{\circ}$ ). We did not run a baseline $0^{\circ}$ block (previous judgment research [e.g., Bingham et al., 2001] and a control experiment had suggested that discrimination of $0^{\circ}$ from its neighbors when the displays were not perturbed was trivial). We therefore could not include a baseline level for the perturbation factor in this analysis, because there was no variance to analyze. 

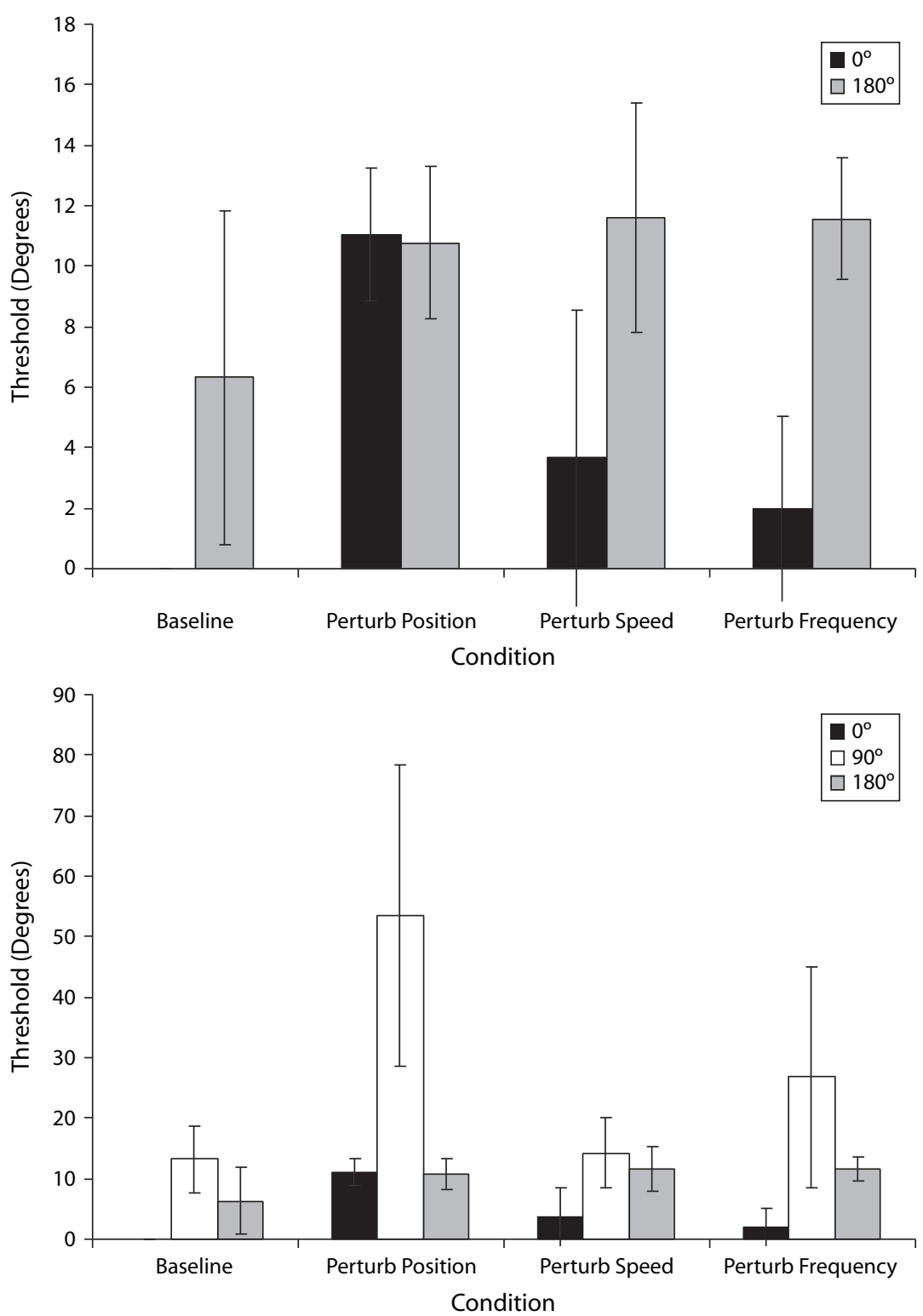

Figure 3. The effect on mean thresholds for all the phase conditions (A: $0^{\circ}$ and $180^{\circ}$ data from Experiment 1; $\mathrm{B}: \mathrm{0}^{\circ}, \mathbf{1 8 0}^{\circ}$, and $90^{\circ}$ data from Experiment 2) and the four perturbation conditions (baseline and perturb position, speed, and frequency). Error bars represent one standard deviation. Note that the baseline 0 data are set to 0 ; see the text for details. The small magnitude of the effects at $0^{\circ}$ and $180^{\circ}$, relative to baseline, confirms that the perturbations did not selectively affect an information variable intrinsic to the perception of these relative phases. In stark contrast, judgments of $90^{\circ}$ were completely disrupted by the position perturbation (note the magnitude of the standard deviation, relative to baseline performance).

Both main effects were statistically reliable [perturbation, $F(2,12)=6.2, p<.05, \eta_{\mathrm{p}}^{2}=.507$; phase, $\left.F(1,6)=26.4, p<.01, \eta_{\mathrm{p}}^{2}=.815\right]$, as was the interaction $\left[F(2,11)=7.7, p<.01, \eta_{\mathrm{p}}^{2}=.563\right]$, which is plotted in Figure 3A. We probed the interaction with 2 one-factor repeated measures ANOVAs (comparing the three perturbation conditions from each phase condition with each other). In the choose $0^{\circ}$ condition, there was a reliable main effect of perturbation $[F(2,12)=12.4$, $\left.p<.01, \eta_{\mathrm{p}}^{2}=.675\right]$, but planned pairwise comparisons (using the Bonferroni adjustment for multiple comparisons) revealed that the effect was caused by thresholds in the position condition being higher than those in all the other conditions (all $p$ s for comparisons with position $<.01$ ), whereas no other conditions differed from each other. 
In the choose $180^{\circ}$ condition, the effect of perturbation was not statistically reliable $\left[F(2,12)=0.147, p=.87, \eta_{\mathrm{p}}^{2}=\right.$ $.024]$. We repeated this last analysis and included the baseline $180^{\circ}$ data; the effect of perturbation was now almost statistically reliable $\left[F(3,18)=3.09, p=.053, \eta_{\mathrm{p}}^{2}=.341\right]$, suggesting that the perturbations had a small but reliable effect on thresholds, relative to baseline performance.

The majority of the group were not truly perturbed by any of the manipulations. There were, however, 3 participants who were selectively and substantially perturbed in the position condition and were, therefore, relying on relative position to perform their judgments. These participants were qualitatively different from the others. This fact tells us several important things. The fact that they were using relative position at both $0^{\circ}$ and $180^{\circ}$ confirms that it is, in fact, possible to do so. This accords nicely with ecological perception-action accounts of perceptual learning, in which a person is attuned to a variable that is sufficiently invariant (by virtue of arising lawfully from the current task environment) to be available long enough for learning to occur (Gibson, 1969; Gibson \& Pick, 2000). In the present task space, clearly, there are two sufficiently invariant variables (relative position and relative direction), and different people, with different developmental histories, can arrive at either one. We can see that relative position is sufficiently informative, because all 3 performed at the same level as the other 7 participants in all the other conditions. This last fact confirms that the position perturbation does selectively affect only relative position; any other consequences would have shown up in the other conditions. Finally, it emphasizes the lesson that individual variability must be taken into account (e.g., Jacobs \& Michaels, 2001), especially when performance is studied in complex task spaces that contain more than one potential solution, which, in turn, emphasizes the need to have a clear delineation of such task spaces so that results such as these are not simply treated as noise.

For the majority of observers, the predictions made by the phase-driven model were supported by Experiment 1 . The next question was what happens after a novel coordination is learned. We used the perturbation paradigm to investigate this.

\section{EXPERIMENT 2 Perturb 90}

As was noted in the introduction, the perception of $90^{\circ}$ is highly variable. This perceptual variability is strongly implicated as the reason why movement at $90^{\circ}$ is variable (Bingham, 2004a, 2004b). The practical upshot of this fact for the present study was that in order to use this paradigm at $90^{\circ}$, we required trained observers of $90^{\circ}$. We had 8 such observers from another study (Wilson \& Bingham, 2008), in which these participants had been trained to choose $90^{\circ}$ in the $2 \mathrm{AFC}$ design used here. In that study, their thresholds had been reduced from $26.03^{\circ}$ to $13.24^{\circ}$.

One interesting result from Wilson and Bingham (2008) was that although the improved performance in the choose $90^{\circ}$ task led to improved movement stability at $90^{\circ}$, the improvement did not generalize to $180^{\circ}$ in either judg- ment or movement tasks. This result is not uncommon; previous movement learning studies also have shown that improvement at $90^{\circ}$ does not generalize to other phases (e.g., Zanone \& Kelso, 1992a, 1992b, 1997). This encapsulation of $90^{\circ}$ raises the intriguing possibility that learning $90^{\circ}$ entails acquiring a new variable, rather than simply improving discrimination of the variable that is currently being used to poor effect. We have already seen that 3 participants in Experiment 1 used position in their perception of relative phase; using variables other than relative direction is therefore possible, and the present method can be employed to identify the variable being used. Although the phase-driven oscillator model made no specific predictions about which variable would be used in trained performance at $90^{\circ}$, it did suggest that at least one of the current perturbations should selectively affect performance and implicate that variable.

\section{Method}

\section{Participants}

Eight participants who had been previously trained in the perceptual discrimination of $90^{\circ}$ returned 3-4 weeks after that study to participate in the perturbation experiments. Four ( 3 of them female, 1 male, from Indiana University) were paid \$10 for each hour-long session; the other 4 (3 of them female, 1 male, from Aberdeen University) were unpaid volunteers. The experiment was again cleared by the local IRB and was performed in accordance with the Declaration of Helsinki.

\section{Procedure}

Choose $90^{\circ}$. The choose $90^{\circ}$ task was identical in design to that used in previous tests. Again, four different locations were tested (two less than $90^{\circ}$, two greater than $90^{\circ}$ ). The different locations were $60^{\circ}$, $75^{\circ}, 105^{\circ}$, and $120^{\circ}$. This set was chosen to be hard, but not at the limit of posttraining performance. Baseline performance was taken as the final posttraining data from Wilson and Bingham (2008), where the participants had judged a block of unperturbed $90^{\circ}$ displays. Both the number of trials for each block and the data analysis were identical to those in Experiment 1. The thresholds for 2 participants in the position perturbation were replaced with the mean of the other 6 , because the regression fit was so poor it produced effectively infinite threshold estimates. They were qualitatively the same as those for the other participants, however, so we replaced their data, rather than remove them (as with the 3 participants in Experiment 1).

\section{Results and Discussion}

The results of Experiment 2 showed that all 8 trained observers of $90^{\circ}$ had their performance completely disrupted by the position perturbation, demonstrating that (1) skilled performance at $90^{\circ}$ does entail using an information variable different from that at $0^{\circ}$ or $180^{\circ}$ and (2) that variable involves relative position. The frequency perturbation also affected performance, but only by about half as much as in the perturb position condition. The implication, however, is that both position and speed are used in the perception of relative phase at $90^{\circ}$ after training.

We performed a repeated measures ANOVA on the mean thresholds for 8 participants. There was one withinsubjects factor, perturbation (four levels: baseline, position, speed, or frequency), which revealed a statistically reliable main effect of perturbation $[F(1.332,9.326)=$ $10.6, p<.01, \eta_{\mathrm{p}}^{2}=.602$; degrees of freedom corrected 
for violation of sphericity, using the Greenhouse-Geisser correction]. Planned pairwise comparisons established that performance with both the position and the frequency manipulations was significantly different from the baseline levels $(p<.01)$ and from that shown in the speed data $(p<.05)$, which did not differ from baseline. Nothing else was significant. The effect is plotted in Figure 3B.

It seems clear from Figure 3B that an actual perturbation effect of the position condition is specific to $90^{\circ}$. To demonstrate this, we compared the Experiment 2 data with the $0^{\circ}$ and $180^{\circ}$ data from Experiment 1 separately (there was no way to do the analysis on all three conditions simultaneously). We performed two mixed design ANOVAs with perturbation (three levels) as a within-subjects factor and phase (two levels: either $0^{\circ}$ and $90^{\circ}$ or $180^{\circ}$ and $90^{\circ}$ ) as a between-subjects factor. Both ANOVAs showed two significant main effects, qualified by a significant interaction between phase and perturbation: $90^{\circ}$ versus $0^{\circ}$ [perturbation, $F(2,26)=10.7, p<.01, \eta_{\mathrm{p}}^{2}=.452$; phase, $F(1,13)=$ 54.6, $p<.01, \eta_{\mathrm{p}}^{2}=.808$; perturbation $\times$ phase, $F(2,26)=$ $4.59, p<.05, \eta_{\mathrm{p}}^{2}=.261$ ]; $90^{\circ}$ versus $180^{\circ}$ [perturbation, $F(2,26)=6.9, p<.01, \eta_{\mathrm{p}}^{2}=.349 ;$ phase, $F(1,13)=33.9$, $p<.01, \eta_{\mathrm{p}}^{2}=.723$; perturbation $\times$ phase, $F(2,26)=7.6$, $\left.p<.01, \eta_{\mathrm{p}}^{2}=.370\right]$. The two interactions (and using the single-factor analyses as post hoc) confirm that thresholds at $90^{\circ}$ were significantly higher than those at $0^{\circ}$ or $180^{\circ}$ for both the position and frequency perturbations. ${ }^{2}$

\section{GENERAL DISCUSSION}

In the present experiments, we investigated what perceptual information underlies judgments about coordinated rhythmic movement in both untrained (at $0^{\circ}$ and $180^{\circ}$, the two intrinsically stable states) and trained (at $90^{\circ}$ ) observers. We employed a novel perturbation method to identify whether any of the candidate perceptual variables suggested by the phase-driven oscillator model (Bingham, 2001, 2004a, 2004b) fit into the composition and organization of the overall task dynamic. Three participants in Experiment 1 were shown to be using relative position at $0^{\circ}$ and $180^{\circ}$, but the majority of the participants were not significantly affected by any of the perturbations, ruling out all other candidate variables and leaving relative direction as the only remaining source of information. The latter result was consistent with the predictions of the model. All expert observers of $90^{\circ}$ in Experiment 2 were completely perturbed by the position perturbation, demonstrating that the process of improving perceptual discrimination at $90^{\circ}$ entails learning a new information variable - specifically, relative position. Perturbations of speed and frequency, which preserved the spatial reference frame but perturbed the value of peak relative speed, added noise to their performance.

The evidence supporting relative direction's necessary role in the perception of relative phase is unambiguous. We attempted to perturb relative direction independently of mean relative phase in pilot work, and this proved impossible, as was described in the introduction. This work, combined with all the previous behavioral evidence re- viewed above and the fact that 7 participants in Experiment 1 were unaffected by any perturbations of the only other candidate variables, made it clear that relative direction plays a foundational role in creating the task space and that perturbing relative direction without violating the boundaries of the coordinated rhythmic movement task space is impossible. Relative direction must be uniquely definable for the coordination phenomena to emerge and is, hence, fundamental to the very definition of the task space. This is also true of the movement task (Wilson et al., 2005a).

The present results suggest that the phase-driven oscillator model (Bingham, 2001, 2004a, 2004b) is incomplete or, more specifically, limited to modeling untrained observers. Although it successfully describes the information underlying performance at $0^{\circ}$ and $180^{\circ}$ for the majority of observers, it is unable to account for the performance of trained $90^{\circ}$ observers. This is unsurprising, because the model was designed to capture the untrained coordination pattern and it, therefore, produces highly variable performance at $90^{\circ}$ in simulations of both judgment and movement by design. Posttraining, the participants in Experiment 2 no longer exhibited the pattern in either movement or perceptual stability (Wilson \& Bingham, 2008). Nonetheless, the manipulations inspired by the model led to uncovering the result in Experiment 2. It is clear that the information used to simulate expert judgments of coordinated movements at $90^{\circ}$ must incorporate relative position, as well as relative direction. Further work is required (and is ongoing) to expand the model to account for learning.

One consistent feature of learning $90^{\circ}$ is the fact that this training only ever generalizes to the symmetry partner, with no improvement at, for instance, $180^{\circ}$ (Zanone \& Kelso, 1992a, 1992b, 1997). The present data suggest that the reason for this encapsulation is that learning $90^{\circ}$ entails learning a novel information variable that is not used at $0^{\circ}$ or $180^{\circ}$ - specifically, one involving relative position, rather than relative direction. Why did the participants attune to relative position in particular? Discriminating $90^{\circ}$ essentially requires one to detect that the endpoint of one oscillator is aligned in time with the midpoint (and location of peak speed) of the other (and vice versa). It is, therefore, something that depends on the perception of both the location of peak speed and its magnitude (the latter identifying that it is, indeed, peak speed). The two perturbations that affected performance are related along these lines. The position perturbation ${ }^{3}$ removed both the reference frame and the value of peak speed, whereas the frequency perturbation only affected peak speed; observers could see where they were with respect to their position, relative to the endpoints and midpoint, but not with respect to the peak speed. In other words, the position perturbation was spatial, whereas the frequency perturbation was temporal, and that of the latter was about half that of the former. The pattern of data suggests that, perceptually, the frequency perturbation added noise to the displays, which affected detection of the information required to perform the judgment but did not eliminate the information itself. 
These results suggest that to judge $90^{\circ}$, participants learn to detect when position endpoints are aligned with peak velocity and vice versa. This alignment is specific to $90^{\circ}$ and does not generalize to any other relative phase, and this is one possible reason why the information (and hence, the learning) does not generalize to $0^{\circ}$ and $180^{\circ}$ (e.g., as can be seen in Zanone \& Kelso, 1992a, 1992b, 1997). However, once one has learned to align positions and/or peak velocities, one could, in principle, just use the alignment of position endpoints, together with alignment of either same-direction peak velocities (to judge $0^{\circ}$ ) or opposite-direction peak velocities (to judge $180^{\circ}$ ). However, if participants were to do this, note that they still would also have to deal in direction; thus, the perception of relative phase at $0^{\circ}$ and $180^{\circ}$ would now entail the perception of three properties (position, speed, and direction), rather than only one (direction). Furthermore, aligning these discrete locations is likely to be less stable than continuously perceiving relative direction, which is available at every moment of time, so there would be no drive to switch from a previously learned variable. There were 3 participants in Experiment 1 who used this information at $0^{\circ}$ and $180^{\circ}$, however, and their unperturbed performance was equivalent to that of the other participants; it remains to be seen how they would be affected by an increase in task difficulty (scaling frequency, for instance).

Another interesting possibility opens up, given the presence of those 3 participants; perhaps participants such as these would already be able to do $90^{\circ}$ or, maybe, show an advantage in learning to perform $90^{\circ}$. They may also not demonstrate this partitioning of the space, showing greater generalization of learning. Clearly, there are several questions that arise from the present data, but the perturbation paradigm described here can be used to find the answers.

Perceptual information is an integral part of the organization of any perception-action dynamical system. In this project, we set out to investigate the identity and role of perceptual information in the long-studied rhythmic movement coordination task dynamic, using a novel perturbation paradigm. The results support the analysis of this task as fundamentally a perception-action task and also support the analysis of such a perception-action task as a dynamical system, whose composition and organization can be explored via perturbation methods. In a dynamical systems approach, if perturbing a given component requires the (functional) disassembly of the system under study, that component can be sensibly thought of as necessary. Relative direction was impervious to perturbation. At $0^{\circ}$ and $180^{\circ}$, most observers were unaffected by perturbations of the other candidate variables. This is strong evidence that relative direction is the necessary component for the formation of a rhythmic movement coordination perception-action system and that it is the variable that specifies mean relative phase. Trained $90^{\circ}$ observers switch to using their learned secondary variable, relative position, but only at $90^{\circ}$, when that information can improve performance. The system is flexible and driven to produce stable behavior.

\section{AUTHOR NOTE}

Experiment 2 was done as part of the first author's dissertation while at Indiana University. A.D.W. thanks his committee for their time, work, and comments. The authors also thank Kathleen Menges for her help in data collection for Experiment 1. Correspondence concerning this article should be addressed to A. D. Wilson, School of Psychology, College of Life Sciences and Medicine, University of Aberdeen, Aberdeen AB24 2UB, Scotland (e-mail: andrew.wilson@abdn.ac.uk).

\section{REFERENCES}

Bingham, G. P. (1988). Task-specific devices and the perceptual bottleneck. Human Movement Science, 7, 225-264.

Bingham, G. P. (2001). A perceptually driven dynamical model of rhythmic limb movement and bimanual coordination. In J. D. Moore $\&$ K. Stenning (Eds.), Proceedings of the 23rd Annual Conference of the Cognitive Science Society (pp. 75-79). Mahwah, NJ: Erlbaum.

Bingham, G. P. (2004a). Another timing variable composed of state variables: Phase perception and phase driven oscillators. In H. Hecht \& G. J. P. Savelsbergh (Eds.), Time-to-contact (Advances in Psychology, Vol. 135, pp. 421-442). Amsterdam: Elsevier.

BingHAM, G. P. (2004b). A perceptually driven dynamical model of bimanual rhythmic movement (and phase perception). Ecological Psychology, 16, 45-53.

Bingham, G. P., Schmidt, R. C., \& ZaAL, F. T. J. M. (1999). Visual perception of relative phasing of human limb movements. Perception \& Psychophysics, 61, 246-258

Bingham, G. P., ZaAl, F. T. J. M., Shull, J. A., \& Collins, D. (2001). The effect of frequency on visual perception of relative phase and phase variability of two oscillating objects. Experimental Brain Research, 136, 543-552.

Bogaerts, H. [P.], Buekers, M. J., ZaAl, F. T. [J. M.], \& Swinnen, S. P. (2003). When visuo-motor incongruence aids motor performance: The effect of perceiving motion structures during transformed visual feedback on bimanual coordination. Behavioural Brain Research, 138, 45-57.

Braunstein, M. L., Hoffman, D. D., \& Pollick, F. E. (1990). Discriminating rigid from nonrigid motion: Minimum points and views. Perception \& Psychophysics, 47, 205-214.

Buekers, M. J., Bogaerts, H. P., Swinnen, S. P., \& Helsen, W. F. (2000). The synchronization of human arm movements to external events. Neuroscience Letters, 290, 181-184.

Fontaine, R. J., Lee, T. D., \& Swinnen, S. P. (1997). Learning a new bimanual coordination pattern: Reciprocal influences of intrinsic and to-be-learned patterns. Canadian Journal of Experimental Psychology, 51, 1-9.

Gibson, E. J. (1969). Principles of perceptual learning and development. New York: Appleton-Century-Crofts.

Gibson, E. J., \& Pick, A. D. (2000). An ecological approach to perceptual learning and development. Oxford: Oxford University Press.

Haken, H., Kelso, J. A. S., \& Bunz, H. (1985). A theoretical model of phase transitions in human hand movements. Biological Cybernetics, $\mathbf{5 1}, 347-356$

Jacobs, D. M., \& Michaels, C. F. (2001). Individual differences and the use of nonspecifying variables in learning to perceive distance and size: Comments on McConnell, Muchisky, and Bingham (1998). Perception \& Psychophysics, 63, 563-571.

Johansson, G. (1950). Configurations in event perception. Uppsala, Sweden: Almqvist \& Wiksell.

Kelso, J. A. S., \& Zanone, P. G. (2002). Coordination dynamics of learning and transfer across different effector systems. Journal of Experimental Psychology: Human Perception \& Performance, 28, 776-797.

Mechsner, F., Kerzel, D., Knoblich, G., \& Prinz, W. (2001). Perceptual basis of bimanual coordination. Nature, 414, 69-73.

Mechsner, F., \& KNoblich, G. (2004). Do muscles matter for coordinated action? Journal of Experimental Psychology: Human Perception \& Performance, 30, 490-503.

Muchisky, M. M., \& Bingham, G. P. (2002). Trajectory forms as a source of information about events. Perception \& Psychophysics, 64, 15-31.

Schmidt, R. C., Carello, C., \& Turvey, M. T. (1990). Phase transi- 
tions and critical fluctuations in the visual coordination of rhythmic movements between people. Journal of Experimental Psychology: Human Perception \& Performance, 16, 227-247.

Temprado, J. J., Swinnen, S. P., Carson, R. G., Tourment, A., \& LauRENT, M. (2003). Interaction of directional, neuromuscular and egocentric constraints on the stability of preferred bimanual coordination patterns. Human Movement Science, 22, 339-363.

Wenderoth, N., Bock, O., \& Krohn, R. (2002). Learning a new bimanual coordination pattern is influenced by existing attractors. Motor Control, 6, 166-182.

Wickelgren, E. A., \& Bingham, G. P. (2001). Infant sensitivity to trajectory forms. Journal of Experimental Psychology: Human Perception \& Performance, 27, 942-952.

Wickelgren, E. A., \& Bingham, G. P. (2004). Perspective distortion of trajectory forms and perceptual constancy in visual event identification. Perception \& Psychophysics, 66, 629-641.

Wickelgren, E. A., \& Bingham, G. P. (2008). Trajectory forms as information for visual event recognition: $3-D$ perspectives on path shape and speed profile. Perception \& Psychophysics, 70, 266-278.

Wilson, A. D., \& Bingham, G. P. (2008). Improved perception leads (eventually) to improved movement stability. Manuscript submitted for publication.

Wilson, A. D., Bingham, G. P., \& Craig, J. C. (2003). Proprioceptive perception of phase variability. Journal of Experimental Psychology: Human Perception \& Performance, 29, 1179-1190.

Wilson, A. D., Collins, D. R., \& Bingham, G. P. (2005a). Human movement coordination implicates relative direction as the information for relative phase. Experimental Brain Research, 165, 351-361.

Wilson, A. D., Collins, D. R., \& Bingham, G. P. (2005b). Perceptual coupling in rhythmic movement coordination: Stable perception leads to stable action. Experimental Brain Research, 164, 517-528.

Wimmers, R. H., Beek, P. J., \& van Wieringen, P. C. W. (1992). Phase transitions in rhythmic tracking movements: A case of unilateral coupling. Human Movement Science, 11, 217-226.

ZaAl, F. T. J. M., Bingham, G. P., \& Schmidt, R. C. (2000). Visual perception of mean relative phase and phase variability. Journal of Experimental Psychology: Human Perception \& Performance, 26, 1209-1220.

Zanone, P. G., \& Kelso, J. A. S. (1992a). Evolution of behavioral attractors with learning: Nonequilibrium phase transitions. Journal of Experimental Psychology: Human Perception \& Performance, 18, 403-421.
Zanone, P. G., \& Kelso, J. A. S. (1992b). Learning and transfer as dynamical paradigms for behavioral change. In G. E. Stelmach \& J. Requin (Eds.), Tutorials in motor behavior II (Advances in Psychology, Vol. 87, pp. 563-581). Amsterdam: North-Holland.

Zanone, P. G., \& Kelso, J. A. S. (1994). The coordination dynamics of learning: Theoretical structure and experimental agenda. In S. P. Swinnen, H. Heuer, J. Massion, \& P. Casaer (Eds.), Interlimb coordination: Neural, dynamical, and cognitive constraints (pp. 461-490). San Diego: Academic Press.

Zanone, P. G., \& Kelso, J. A. S. (1997). Coordination dynamics of learning and transfer: Collective and component levels. Journal of Experimental Psychology: Human Perception \& Performance, 23, 1454-1480.

\section{NOTES}

1. It seems clear that the causation works this way around; if perception is stable because the movements are easy, making the movements easy should improve perception. This is not the case (see Wilson et al., 2003, for a detailed discussion of this point).

2 . We thank an anonymous reviewer for suggesting these analyses.

3. One potential issue with the position perturbation condition is the fact that, unlike the other perturbations, the changes were random. It is plausible that the effect described here was due to randomness, rather than being a specific information perturbation. There are two reasons why we feel this is not an issue, however. First, analysis of the time series (i.e., the signal to be perceived; Figure 1) clearly showed that relative direction and, hence, relative phase were defined correctly at all times. The information was, therefore, in principle, available, if relative direction was the information in question. This leads to the second reason, which was that perturbation had, at most, a minor effect at $0^{\circ}$ or $180^{\circ}$. Although the effect at $0^{\circ}$ was statistically significant, it was nearly six times smaller than the effect at $90^{\circ}$, suggesting that the lack of rigid motion only made $0^{\circ}$ slightly harder. These two points (analysis of the signal and the fact that the perturbation was effectively restricted to $90^{\circ}$ ) suggest that the randomness element per se does not account for the effect. The position perturbation therefore selectively perturbed the information used to judge $90^{\circ}$.

(Manuscript received April 2, 2007; revision accepted for publication October 16, 2007.) 